DOI: $10.4274 /$ jarem.galenos.2018.2659

J Acad Res Med 2020; 10(1): 23-6

\title{
The Effect of Smoking on Serum Nesfatin-1 Levels on Women Who are in Follicular Phase: A Pilot Study
}

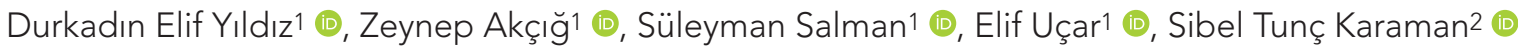 \\ ${ }^{1}$ University of Health Sciences Turkey, Gaziosmanpaşa Training and Research Hospital, Clinic of Obstetrics and Gynecology, İstanbul, Turkey \\ 2University of Health Sciences Turkey, Gaziosmanpaşa Training and Research Hospital, Clinic of Family Medicine, İstanbul, Turkey
}

Cite this article as: Yıldız DE, Akçığ Z, Salman S, Uçar E, Tunç Karaman S. The Effect of Smoking on Serum Nesfatin-1 Levels on Women Who are in Follicular Phase: A Pilot Study. JAREM 2020;10(1): 23-6

\begin{abstract}
Objective: Nesfatin-1, derived from NEFA/nucleobindin 2, is a hormone that suppresses food intake via melanocortin system in hypothalamus and has role on ovarian development. In this study, we aimed to determine the effect of smoking on serum nesfatin-1 levels on women who were in early follicular phase.

Methods: This study included 32 female participants, as smokers $(n=11)$ and non-smokers $(n=21)$. Nesfatin-1, fasting blood glucose, low density lipoprotein (LDL), high density lipoprotein (HDL), total cholesterol and tryglicerides, follicule stimulating hormone (FSH), luteinizing hormone (LH), antimüllerian hormone (AMH) and estradiol levels were measured from the blood samples in early follicular phase and they were compared.

Results: $34,3 \%(n=11)$ of participants were smokers and according to the Fagerström Nicotine Dependence Scale whose nicotine dependence levels were $>7$ while $65.6 \%(n=21)$ were non-smokers. Serum nesfatin-1 levels in smokers were $(81 \pm 0.1 \mathrm{pg} / \mathrm{mL})$ found significantly lower than non-smokers $(125 \pm 0.1 \mathrm{pg} / \mathrm{mL})(\mathrm{p}=0.007)$. No significant differences were found between these two groups about the levels of fasting blood glucose, LDL, HDL, total cholesterol, triglycerides, FSH, LH, AMH and estradiol.

Conclusion: The levels of serum nesfatin-1 was found significantly lower in female smokers than the non-smokers in early follicular phase.

Keywords: Nesfatin-1, smoking, follicular, nicotine, ovarian reserve
\end{abstract}

\section{INTRODUCTION}

Obesity causes important health problems in our country and in the world. Due to the rapidly increasing obesity rates in the society, many studies are conducted to understand the biochemical mechanisms that govern our dietary behavior. Nesfatin-1 precursor protein, the first identified effect of which is to suppress appetite, is a polypeptide consisting of 82 amino acids synthesized from nucleobindin (NUCB-2) (1). Nicotine, which is responsible for most of the metabolic effects of smoking, also suppresses appetite, such as nesfatin-1 (2), and takes part in energy metabolism (3).

In addition to suppressing appetite, it has been shown in animal studies that it slows gastric emptying (4), increases glucose- stimulated insulin release and sensitivity (5), lowers lipid levels in blood and suppresses lipogenesis (6). In different studies nesfatin-1 levels have been shown to vary significantly in pregnant women (7), women who are breastfeeding (8), women with menstrual irregularities (21-35 days/2-7 days/a part from women menstruating with 3-4 pads per day) (2); in women with ovarian cyst (3), endometriosis (9), myoma uterus (10), polycystic ovarian syndrome (11), endocrine disease [diabetes mellitus (DM), Cushing disease, adrenal diseases, diseases of the thyroid gland, prolactinoma] (12), epilepsy (13) sleep apnea (14), depression (15), liver and kidney failure (16), and hypertension (17); gastrointestinal system diseases including dyspepsia and previous gastrointestinal

ORCID IDs of the authors: D.E.Y. 0000-0001-8396-5542; Z.A. 0000-0002-5719-3910; S.S. 0000-0001-7090-6105; E.U. 0000-0001-5302-4688;

S.T.K. 0000-0003-1833-8758. 
surgery history (18), women with previous ovarian surgery history and suspicion of malignancy (19); women (20) who use medication, including oral contraceptives, and women who regularly exercise intensely (3 times a week and more than 30 minutes) (21). The saturation molecule nesfatin-1 also takes part in the triggering of puberty and in the development of ovaries (22). It has been shown to be elevated in polycystic ovarian patients and is thought to affect the hypothalamus-pituitary-ovarian axis (23). This suggested that the nesfatin-1 molecule had a role in gonadal development.

Numerous studies have been conducted in the literature that reveal the relationship between nesfatin-1 levels and many biochemical parameters. Nesfatin-1 has been related with ovarian functions, and ovarian diseases and nesfatin-1 levels have been associated. However, in the literature review we have made we have not found a publication about how smoking changes nesfatin-1 levels. Predicting that intensive nicotine stimulation in smokers will change the levels of nesfatin in the blood, we measured serum nesfatin-1 levels in the early follicular phase in the $3^{\text {rd }}$ day of menstruation, along with ovarian reserve tests, in women who smoke and do not smoke.

Our primary goal in this study is to determine how smoking affects nesfatin-1 levels. Our secondary goal is to compare the ovarian reserve tests and nesfatin-1 levels to reveal the relationship between them.

\section{METHODS}

This study included women who applied to University of Health Sciences Turkey Gaziosmanpaşa Training and Research Hospital, Gynecology and Obstetrics Clinic policlinics and Smoking Cessation policlinic between 15 August and 30 October 2018. A signed informed consent form was obtained from each woman participating in the study. Anamnesis of each volunteer was taken and a physical examination was performed in order to determine whether the volunteer individuals had an obstacle to the study. The age, height and weight information obtained were recorded and body mass indexes (BMI) were calculated. Women of childbearing age (18-45 years) were included in the study. Women with pregnancies, breastfeeding women, menstrual irregularities (21-35 days/2-7 days/apart from women who menstruate 3-4 pads daily); women with ovarian cyst, endometriosis, myoma uterus, polycystic ovarian syndrome, endocrine disease (DM, Cushing disease, adrenal diseases, diseases of the thyroid gland, prolactinoma), epilepsy, sleep apnea, depression, liver and kidney failure, hypertension; women with gastrointestinal system diseases including dyspepsia and previous history of gastrointestinal surgery, history of previous ovarian surgery, and suspicion of malignancy; Women with drug use, including oral contraceptives, and women who regularly exercise intensely (3 times a week and more than 30 minutes) were not included. The exclusion criteria were created with the cases whose effect on nesfatin-1 was determined by searching the literature.

Volunteers were divided into two groups as smokers and nonsmokers. According to the Fagerstörm nicotine addiction test, women with a high level of smoking dependence $(\geq 7)$ were included in the smoker group, and volunteers with low test results were excluded from the study. Eleven smokers and 21 nonsmoker volunteers participated in this preliminary study. Venous blood samples were taken from all volunteers in the early follicular phase of the menstrual cycle ( $3^{\text {rd }}-4^{\text {th }}$ day of menstruation) between 10.00-11.00 after 8-12 hours of fasting. Blood glucose and blood lipid measurements [total cholesterol, low density lipoprotein $(\mathrm{LDL})$, high density lipoprotein (HDL), triglycerides] were taken from the samples and ovarian function tests [follicule stimulating hormone (FSH), luteinizing hormone (LH), anti-Müllerian hormone $(\mathrm{AMH})$, Estradiol] were studied. Nesfatin-1 levels from samples were measured by ELISA method with commercial kits (Phoenix Pharmaceutical, INC, California, USA).

All procedures were carried out in accordance with the Helsinki declaration. Before starting the study, necessary permission was obtained from the Gaziosmanpaşa Training and Research Hospital Ethics Committee (approval number: 72, date: 08.08.2018).

\section{Statistical Analysis}

The normality control of demographic data and blood measurements was done by drawing the Shapiro-Wilk test, histogram, Q-O plot and box plot charts. Data were given as median, minimum and maximum. The measurement variables between the two groups were analyzed with the Mann-Whitney $U$ test. The limit of significance was taken as $p<0.05$ and bidirectional. The analyzes were done using NCSS 10 (2015. Kaysville, Utah, USA) software program.

\section{RESULTS}

While $34.3 \%(n=11)$ of the study participants were smokers and high-level ( $\geq 7$ ) cigarette addict women according to Fagerstörm nicotine addiction test, $65.7 \%(n=21)$ were non-smoker women. All of the volunteers were women of childbearing age between the ages of 18-45, and their mean age was 31.5. The median value of the non-smoker group was 27 and the smoker group was 33, and there was no statistically significant difference between the two groups. While the BMI median value in the smoker group was 26.2, it was 22.7 in the smoker group. Considering the fertility data of the volunteers who participated in the study, 13 of 32 women were women who had never experienced a pregnancy (gravida: 0 ), and 3 were grand multipa (gravida $\geq 5$ ). The demographic characteristics of both groups are given in the table (Table 1).

There was no significant difference in the evaluation in terms of fasting blood glucose, LDL, HDL, total cholesterol, triglyceride, $\mathrm{FSH}, \mathrm{LH}, \mathrm{AMH}$ and estradiol levels from blood samples taken from both volunteer groups ( $p>0.05$ ) (Table 2).

Serum nesfatin-1 levels were significantly lower in the smoker group (median value: $81 \pm 0.1 \mathrm{pg} / \mathrm{mL}$ ) compared to the non-smoker group (median value: $125 \pm 0.1 \mathrm{pg} / \mathrm{mL})(p=0.007)$.

\section{DISCUSSION}

In a study conducted by Kim et al. (24), saturation molecule nesfatin-1 was detected in teka and interstitial cells in mouse 


\begin{tabular}{|c|c|c|c|}
\hline \multirow{2}{*}{ Variables } & \multicolumn{2}{|c|}{ Median value (minimum-maximum) } & \multirow{2}{*}{$p^{*}$} \\
\hline & In non-smokers & In smokers & \\
\hline Age (year) & $27(18-44)$ & $33(21-45)$ & 0.099 \\
\hline $\begin{array}{l}\text { Body mass index } \\
\left(\mathrm{kg} / \mathrm{m}^{2}\right)\end{array}$ & $26.2(19.1-33.9)$ & $22.7(18.8-39.1)$ & 0.293 \\
\hline Gravida & $1(0-5)$ & $2(0-5)$ & 0.308 \\
\hline Parity & $1(0-5)$ & $2(0-5)$ & 0.439 \\
\hline
\end{tabular}

\section{Table 2. Results of smoking and non-smoking group}

\begin{tabular}{|c|c|c|c|}
\hline \multirow{2}{*}{ Variables } & \multicolumn{2}{|c|}{ Median value (minimum-maximum) } & \multirow{2}{*}{$p^{*}$} \\
\hline & In non-smokers & In smokers & \\
\hline $\begin{array}{l}\text { Fasting blood } \\
\text { glucose (mg/dL) }\end{array}$ & $83(64-92)$ & $85(64-106)$ & 0.551 \\
\hline LDL (mg/dL) & $102(33-168)$ & $109(53-120)$ & 0.662 \\
\hline $\mathrm{HDL}(\mathrm{mg} / \mathrm{dL})$ & $48(35-78)$ & $43(36-111)$ & 0.606 \\
\hline $\begin{array}{l}\text { Total cholesterol } \\
\text { (mg/dL) }\end{array}$ & $167(87-263)$ & $170(104-192)$ & 0.691 \\
\hline Triglyceride (mg/dL) & $77(28-232)$ & $77(35-217)$ & 0.606 \\
\hline $\mathrm{FSH}(\mathrm{mlU} / \mathrm{mL})$ & $6.7(4,1-9.6)$ & $6(5-72)$ & 0.331 \\
\hline $\mathrm{LH}(\mathrm{mIU} / \mathrm{mL})$ & $4.9(2-9.9)$ & $6(2-41)$ & 0.383 \\
\hline Estradiol (pg/mL) & $47(20-174)$ & $66(20-253)$ & 0.565 \\
\hline $\mathrm{AMH}(\mathrm{ng} / \mathrm{mL})$ & $2.2(0.6-4.8)$ & $2(0-6)$ & 0.19 \\
\hline Nesfatin-1 (pg/mL) & $125.2(48.7-3098.6)$ & $81(46-235)$ & 0.007 \\
\hline
\end{tabular}

*Mann-Whitney U Test, LDL: low density lipoprotein, HDL: high density lipoprotein, FSH: follicle-stimulating hormone, LH: luteinizing hormone, $\mathrm{AMH}$ : anti-mullerian hormone

ovarian tissue. Nesfatin-1 is also involved in triggering puberty and in the development of ovaries. It has been shown to be elevated in patients with polycystic ovaries (13) and is thought to affect the hypothalamus-pituitary-ovarian axis. This suggested that the nesfatin-1 molecule had a role in gonadal development.

The increase in pubertal transition period and premature telarchy supports the role of nesfatin-1 molecule in gonadal development (25). In another study conducted in rats, it was observed that when nasfatin-1 was given intravenously, gonadotropins increased and when antidote was given, ovarian volume decreased, which is a symptom of delayed puberty (7). This information suggests that the nesfatin-1 molecule can give an idea about the fertility age female ovarian reserve. In our study, we compared nesfatin-1 levels and basal FSH, estradiol, and AMH levels, which were measured on the third day of menstruation with ovarian reserve markers.

In our study, $\mathrm{AMH}$ values showing ovarian reserve were found to be lower in the smoker group, although not statistically significant. In addition, nesfatin-1 values were significantly lower in the smoker group. This result may be due to the relative deficiency of our number of cases.

In the study of 477 women of childbearing age examined by Kline et al. (26); it has been determined that there is an increase of approximately $15 \%$ in FSH values in women who smoke. In our study, although FSH levels were found to be increased in the smoker group, the difference was not statistically significant. In the same study, AMH values were found to be decreased in the smoker group. However, the difference is not statistically significant. In our study, $\mathrm{AMH}$ values were found lower in the smoker group (2.2 vs 2.0, $p=0.19$ ). This may be due to the insufficient number of volunteers in our study.

In a study by Ayada et al. (27), it was shown that nesfatin-1 expression increased in puberty and an increase in $\mathrm{LH}$ was observed in parallel with this increase. In addition, in another study by García-Galiano et al. (22) it was found that high nesfatin-1 levels increased hypothalamic NUCB-2 protein content and serum $\mathrm{LH}$ levels. In parallel with the studies conducted, $\mathrm{LH}$ values in our study were found to be higher in the non-smoking group with higher levels of nesfatin-1 serum, but not statistically.

Another effect of nicotine in cigarette is that it negatively affects sex hormone levels such as plasma testosterone and estrogen (27). In a study conducted by Park et al. (28) to demonstrate the effects of smoking on sex hormone levels and sexual functions, it was found that smoking had a negative effect on testosterone levels as expected in men and caused a significant decrease in sexual functions, but did not cause a significant decrease in estrogen levels in women. However, although there is no significant decrease in blood estrogen levels, it has been reported that there is a loss in sexual functions and cigarette has an antiestrogenic effect in the genital system. In our study, blood estrogen levels were higher in the smoker group.

Multiple studies have been conducted to determine the link between the Nesfatin-1 level and the BMI. In a study conducted by Abaci et al. (29) on children, the serum nesfatin-1 level of obese children was found to be significantly lower than the control group. The study by Ramanjaneya et al. (30) on adults showed that there was a positive correlation between plasma nesfatin-1 levels and BMI in patients with a BMI between 22.3 and $27.67 \mathrm{~kg} /$ $\mathrm{m}^{2}$. In our study, we did not find a statistically significant change between BMI and nesfatin-1 levels.

\section{CONCLUSION}

Serum nesfatin-1 values measured in the early follicular phase were found to be significantly lower in smoking women compared to non-smoking women ( $p=0.007)$.

This study shows the relationship between nesfatin-1 and smoking, but it is not enough to explain the mechanism by which smoking caused a decrease in nesfatin-1 level. More research is needed to explain this relationship.

As a preliminary study, after a sufficient number of volunteers are included in the study, other data that we foresee to be meaningful such as ovarian functions and nesfatin-1 relationship can be accessed along with the decrease in standard deviations. Voluntary recruitment continues for our preliminary study, in which we present the results. After the number of volunteers is increased 
for both groups and sufficient volunteers are reached, the results will be presented again.

Available data on the effect of cigarette addiction and nicotine on nesfatin-1 are very limited. Considering that Nesfatin-1 draws attention as an effective therapeutic agent for various diseases, we think it is important to know more about nesfatin-1 from different perspectives.

Ethics Committee Approval: Before starting the study, necessary permission was obtained from the Gaziosmanpaşa Training and Research Hospital Ethics Committee (approval number: 72, date: 08.08.2018).

Informed Consent: A signed informed consent form was obtained from each woman participating in the study.

Peer-review: Internally peer-reviewed.

Author Contributions: Concept - D.E.Y., Z.A., S.S., E.U., S.T.K.; Design D.E.Y., Z.A., S.S., E.U., S.T.K.; Supervision - D.E.Y., Z.A., S.S., E.U., S.T.K.; Resource - D.E.Y.; Materials - D.E.Y.; Data Collection and/or Processing D.E.Y., E.U.; Analysis and/ or Interpretation - D.E.Y., Z.A.; Literature Search - D.E.Y., Z.A.; Writing Manuscript - D.E.Y.; Critical Reviews - S.S., Z.A.

Conflict of Interest: The authors have no conflict of interest to declare.

Financial Disclosure: The authors declared that this study has received no financial support.

\section{REFERENCES}

1. Oh-I S, Shimizu H, Satoh T, Okada S, Adachi S, Inoue K, et al. Identification of nesfatin-1 as a satiety molecule in the hypothalamus. Nature. 2006; 443: 709-12.

2. Alp E, Görmüş U, Güdücü N, Bozkurt S. Nesfatin-1 levels and metabolic markers in polycystic ovary syndrome. Gynecol Endocrinol 2015;31: 5437.

3. Kim J, Yang $H$. Nesfatin-1 as a new potent regulator in reproductive system. Dev Reprod 2012; 16: 253-64.

4. Stengel, A, Goebel M, Wang L, Rivier J, Kobelt P, Monnikes H, et al. Central nesfatin-1 reduces dark-phase food intake and gastric emptying in rats: differential role of corticotropin-releasing factor2 receptor. Endocrinology 2009; 150, 4911-9.

5. Li Z, Gao L, Tang H, Yin Y, Xiang X, Li Y, et al. Peripheral effects of nesfatin-1 on glucose homeostasis. PLoS One 2013; 8: e71513.

6. Yin Y, Li Z, Gao L, Li Y, Zhao J, Zhang W. AMPK-dependent modulation of hepatic lipid metabolism by nesfatin-1. Mol Cell Endocrinol 2015; 417 : 20-6.

7. Garcés MF, Poveda NE, Sanchez E, Sánchez ÁY, Bravo SB, Vázquez MJ, et al. Regulation of NucB2/Nesfatin-1 throughout rat pregnancy. Physiol Behav 2014; 133: 216-22.

8. Çatlı G, Olgaç Dündar N, Dündar BN. Adipokines in breast milk: an update. J Clin Res Pediatr Endocrinol 2014; 6: 192-201.

9. Şengül Ö, Dilbaz B, Halıcı Z, Ferah I, Çadırcı E, Yılmaz F. Decreased serum nesfatin-1 levels in endometriosis. Eur J Obstet Gynecol Reprod Biol 2014; 177: 34-7.

10. Carranza-Lira S, Romero-Cuadra IA. Frequency of presentation and characteristics of chronic pelvic pain at the gynecologic consultation. Rev Med Inst Mex Seguro Soc 2018; 56: 226-30.

11. Ademoglu EN, Gorar S, Carlıoglu A, Yazıcı H, Dellal FD, Berberoglu Z, et al. Plasma nesfatin-1 levels are increased in patients with polycystic ovary syndrome. J Endocrinol Invest 2014; 37: 715-9.

12. Ramesh N, Gawli K, Pasupulleti VK, Unniappan S. Metabolic and cardiovascular actions of nesfatin-1: implications in health and disease. Curr Pharm Des 2017; 23: 1453-64.

13. Pałasz A, Krzystanek M, Worthington J, Czajkowska B, Kostro K, Wiaderkiewicz R, et al. Nesfatin-1, a unique regulatory neuropeptide of the brain. Neuropeptides 2012; 46: 105-12.

14. Shen $P$, Han $Y$, Cai $B$, Wang $Y$. Decreased levels of serum nesfatin-1 in patients with obstructive sleep apnea syndrome. Sleep Breath 2015; 19 : 515-22.

15. Algul S, Ozcelik $O$. Evaluating the levels of nesfatin-1 and ghrelin hormones in patients with moderate and severe major depressive disorders. Psychiatry Investig 2018; 15: 214-8.

16. Jiang $G$, Wang $M$, Wang $L$, Chen $H$, Chen Z, Guo J, et al. The protective effect of nesfatin-1 against renal ischemia-reperfusion injury in rats. Ren Fail 2015; 37: 882-9.

17. Shaparenko OV, Kravchun PG, Kravchun PP, Kadykova OI. Nesfatin-1 role in remodeling of the left ventricle myocardium in patients with arterial hypertension and obesity. Wiad Lek 2018; 71: 1006-9.

18. Ozcan ATD, Altin CB, Erdogan S, Ergin M, Çiftçi A, Kara H, et al. The effects of desflurane and sevoflurane on nesfatin-1 levels in laparoscopic cholecystectomy: a randomized controlled trial. BMC Anesthesiol 2018; 18: 23.

19. Spyrou N, Avgerinos KI, Mantzoros CS, Dalamaga M. Classic and novel adipocytokines at the intersection of obesity and cancer: diagnostic and therapeutic strategies. Curr Obes Rep 2018; 7: 260-75.

20. Aydin B, Guvenc G, Altinbas B, Niaz N, Yalcin M. Modulation of nesfatin1 -induced cardiovascular effects by the central cholinergic system. Neuropeptides 2018; 70: 9-15.

21. Algul S, Ozdenk C, Ozcelik O. Variations in leptin, nesfatin-1 and irisin levels induced by aerobic exercise in young trained and untrained male subjects. Biol Sport 2017; 34: 339-44.

22. García-Galiano D, Navarro VM, Roa J, Ruiz-Pino F, Sánchez-Garrido MA, Pineda $R$, et al. The anorexigenic neuropeptide, nesfatin-1, is indispensable for normal puberty onset in the female rat. $\mathrm{J}$ Neurosci 2010; 30: 7783-92.

23. Deniz R, Gurates B, Aydin S, Celik H, Sahin I, Baykus Y, et al. Nesfatin-1 and other hormone alterations in polycystic ovary syndrome. Endocrine 2012; 42: 694-9.

24. Kim J, Chung $Y$, Kim H, Im E, Lee H, Yang $H$. The tissue distribution of nesfatin-1/NUCB2 in mouse. Dev Reprod 2014; 18: 301-9.

25. Çatlı G, Anık A, Küme T, Çalan ÖG, Dündar BN, Böber E, et al. Serum nesfatin-1 and leptin levels in non-obese girls with premature thelarche. J Endocrinol Invest. 2015; 38: 909-13.

26. Kline J, Tang A, Levin B. Smoking, alcohol and caffeine in relation to two hormonal indicators of ovarian age during the reproductive years. Maturitas 2016; 92: 115-122.

27. Ayada C, Toru Ü, Korkut Y. Nesfatin-1 and its effects on different systems. Hippokratia 2015; 19: 4-10.

28. Park MG, Ko KW, Oh MM, Bae JH, Kim JJ, Moon du G. Effects of smoking on plasma testosterone level and erectile function in rats. J Sex Med 2012; 9: 472-81.

29. Abaci A, Catli G, Anik A, Kume T, Bober E. The relation of serum nesfatin-1 level with metabolic and clinical parameters in obese and healthy children. Pediatr Diabetes 2013; 14: 189-95.

30. Ramanjaneya M, Chen J, Brown JE, Tripathi G, Hallschmid M, Patel S, et al. Identification of nesfatin-1 in human and murine adipose tissue: a novel depotspecific adipokine with increased levels in obesity. Endocrinology 2010; 151: 3169-80. 